\title{
Two Applications of Group Characters to the Solution of Boundary-Value Problems
}

\author{
E. Stiefel ${ }^{2}$
}

\begin{abstract}
It is shown that the numerical work involved in solving a boundary-value or e.genvalue problem by finite difference methods in a domain with many symmetries can often be reduced by applying the theory of group characters to the group of symmetries of the domain.

In the second part the author considers the problem of solving $\Delta u=0$ in a cube when the prescribed boundary values are invariant under the group of rotations of the cube. In the series representation of the solution in terms of harmonic polynomials only a subset of these polynomials actually occurs, and the theory of group characters facilitates considerably the determination of this subset.
\end{abstract}

It is very well known that representation of groups may be a useful tool in discussing various eigenvalue problems in quantum mechanies. This is due to the fact that boundary-value problems of Schroedinger's equation are to be solved mostly in the whole space or inside a sphere as fundamental region and therefore the high symmetry of those domains can be used.

It is the purpose of this paper to show that group characters sometimes are appropriate also to simplify the numerical computation of boundary-value or eigenvalue problems in more general-shaped domains, in particular if the problem has been translated into the language of difference calculus. It will be sufficient to explain this discussing simple examples.

Let, for instance, $A$ be any plane region and $D$ a differential operator invariant under Euclidean motions or reflections of the plane. More exactly, if $f(x, y)$ is a function in our plane and $M$ a Euclidean rotation, translation, or reflection, then

$$
D(M f)=M(D f),
$$

which is to say that $D$ must commute with any motion $M$. The Laplace operator $\Delta$ and the operator $\Delta \Delta$ of elasticity problems have this property.

In order to solve any given boundary-value problem with respect to the region $A$ and the operator $D$, we may use the difference technique, introducing a square lattice $L$ in $A$ and replacing $f$ by a lattice function (defined only in the lattıce points) and $D$ by a difference operator, which approximates the given differential operator. In the case of a $\Delta$-problem, the corresponding difference operator may be given by figure 1. Relation (1) will be true again in the difference case, provided that by $M$ is understood any motion or reflection transforming the lattice into itself.

Suppose now that the given region $A$ is invariant under a group $G$ of transformations of this type. If $A$ has no symmetry at all, we may use Schwarz's alternating process dividing $A$ into overlapping subregions, each of them having symmetry properties.

1 This work was performed on a National Bureau of Standards contract with the University of California, Los Angeles, Calif, and was sponsored (in part) the University of California, Los

by the Office of Naval Research. nische Hochschule, Zurich, Switzerland.

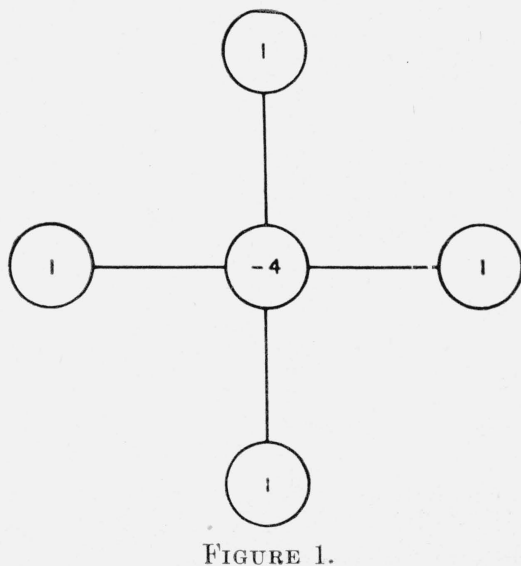

It is our goal to show how the group $G$ can be used in order to simplify the solution of the given boundaryvalue problem in the region $A$.

Take, for instance, the square region $A$ of figure 2 . The group $G$ is in this case the symmetry group of the square of order 8 having the following similarity classes:

\begin{tabular}{|c|c|c|}
\hline Class & Elements & $\begin{array}{l}\text { Number } \\
\text { of } \\
\text { elements }\end{array}$ \\
\hline $\begin{array}{l}{[1] \ldots} \\
{[2] \ldots} \\
{[3]} \\
{[4]_{\ldots}} \\
{[5]_{-\ldots}}\end{array}$ & $\begin{array}{l}\text { Identity } \\
\text { Rotations } \pm 90^{\circ} \\
\text { Rotation } 180^{\circ} \\
\text { Reflection in horizontal or } \\
\text { vertical line } \\
\text { Reflections in diagonal lines }\end{array}$ & $\begin{array}{l}1 \\
2 \\
1 \\
\\
2 \\
2\end{array}$ \\
\hline & Total $\ldots \ldots \ldots$ & 8 \\
\hline
\end{tabular}

The given boundary-value problem is equivalent to a system of nine linear equations for the values of the wanted function in the nine interior lattice points. Any lattice function $f$ may be visualized as a vector in a nine-dimensional vector space $S$. Therefore, applying the operations of the table to the lattice functions, we get a linear representation $R$ of the basic group $G$ in $S$. 


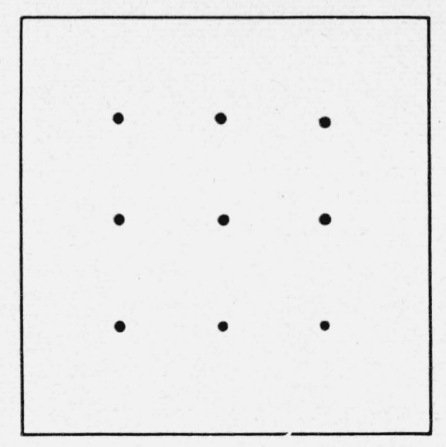

Figure 2.

It is not too hard to show that its character $\chi$ is given by the following rule: The value $\chi(M)$ of $\chi$ for an element $M$ of $G$ is equal to the number of lattice points fixed under the operation $M$. (Use as coordinate system in $S$ the lattice functions not equal to 0 only in one single lattice point; they are permuted by M.) We want to decompose the representation $\dot{R}_{R}$ into irreducible components:

$$
R=c_{1} R_{1}+c_{2} R_{2}+\ldots+c_{5} R_{5},
$$

Where $R_{1}, R_{2}, \ldots, R_{5}$ are the irreducible representations of $G$. (There are exactly five because $G$ has five similarity classes). According to the orthogonality theorems of representation theory this has to be done working on the table of characters of $C$ as follows:

\begin{tabular}{|c|c|c|c|c|c|}
\hline Class _... $\ldots$ & [1] & {$[2]$} & [3] & {$[4]$} & {$[5]$} \\
\hline No. of elements_ & 1 & 2 & 1 & 2 & 2 \\
\hline $\begin{array}{l}\mathrm{X}_{1} \\
\mathrm{X}_{2} \\
\mathrm{X}_{3} \\
\mathrm{X}_{4} \\
\mathrm{X}_{5}\end{array}$ & $\begin{array}{l}1 \\
1 \\
1 \\
1 \\
2\end{array}$ & $\begin{array}{r}1 \\
1 \\
-1 \\
-1 \\
0\end{array}$ & $\begin{array}{r}1 \\
1 \\
1 \\
1 \\
-2\end{array}$ & $\begin{array}{r}1 \\
-1 \\
1 \\
-1 \\
0\end{array}$ & $\begin{array}{r}1 \\
-1 \\
-1 \\
1 \\
0\end{array}$ \\
\hline$\chi$ & 9 & 1 & 1 & 3 & 3 \\
\hline
\end{tabular}

In the first line we have listed the similarity classes with their numbers of elements and in the following lines the values of the characters of the irreducible representations in those classes. They are recorded in many textbooks of representation theory. ${ }^{3}$ The last line is the character $\chi$ of our representation $R$, computed according to the rule previously mentioned. The first number in each line is the degree of the representation (dimension of the vector space transformed by the representation). According to a general formula of character theory the coefficient $c_{i}$ in (2) is

$$
c_{i}=\frac{1}{8} \sum_{(M)} \chi(M) \chi_{i}(M)
$$

\footnotetext{
${ }^{3}$ A. Speiser, Die Theorie der Gruppen von endlicher Ordnung, 3d ed., p. 179 180 (Springer, Berlin; and Dover Pub).
}

with the group element $M$ running through the whole group $G$. By this we have for instance from our table

$$
c_{4}=\frac{1}{8}(9 \cdot 1-2 \cdot 1+1 \cdot 1-2 \cdot 3+2 \cdot 3)=1 .
$$

The multiplicities $c_{i}$ are listed in the column to the right of the table. Finally, we find

$$
R=3 R_{1}+R_{3}+R_{4}+2 R_{5} .
$$

Therefore, the vector space $S$ splits into subspaces

$$
S_{1}, \quad S_{1}^{\prime}, \quad S_{1}^{\prime \prime} ; \quad S_{3} ; \quad S_{4} ; \quad S_{5}, \quad S_{5}^{\prime},
$$

each of them being invariant under $R$ and being transformed by the irreducible representation given by its subscript.

Take now a set of subspaces having the same subscript (i. e., being transformed by the same representation) and introduce base vectors in each space of the set. For instance, $S_{5}$ and $S_{5}{ }^{\prime}$, being both two-dimensional, may be spanned by the base vectors

$$
\begin{array}{lll}
S_{5}: & e_{51}, & e_{52} \\
S_{5}^{\prime}: & e_{51}^{\prime}, & e_{52}^{\prime} .
\end{array}
$$

Generally speaking, each set of equally transformed subspaces yields a rectangular arrangement of base vectors by listing the base vectors of each subspace in a line. The length of the rectangle is the degree of the irreducible representation under consideration and its height the multiplicity of this representation as a component of $R$. It is, of course, possible to choose the base vectors in such a way that each line of the rectangle is transformed exactly in the same way.

It follows then from a well-known generalization ${ }^{4}$ of Schur's lemma that each linear operator in $S$ commutable with the transformations of $R$ transforms the vectors of each column in our rectangle among themselves and in each column in the same way. Taking into account the relation (1), we see that the difference operator of the given boundary-value problem has this property. From this follows immediately that the original system of nine linear equations with nine unknowns splits into partial systems. The rectangle (8), for instance, yields two systems of two equations each, having the same coefficient matrices. From the first and the $c$ column of the table (4) we get therefore the following final result. The system of nine equations splits into one system of three simultaneous equations, one single equation, one single equation, and two systems of two simultaneous equations.

If we have to deal with an eigenvalue problem instead of a boundary-value problem, the analogous statement is, that the wanted characteristic polynomial is the product of a factor of third degree, two linear factors and the square of a quadratic factor. In order to carry out the decomposition of the system of nine equations explicitly, it is, of course, necessary

${ }^{4}$ B. L. van der Waerden, Die gruppentheoretische Methode in der Quantenmechanik, sect. 13 , p. 47-50 (Springer, Berlin, 1932). 
to establish the lattice functions, which build the base vectors of the different rectangles (8). The three basic lattice functions corresponding to the first irreducible representation $R_{1}$ are simply three linear independent functions invariant under all motions of the square. As another example we give the values of the lattice functions (8) in the nine lattice points.

$$
\begin{aligned}
& e_{51}=\begin{array}{rrr}
0 & 0 & 0 \\
-1 & 0 & 1 \\
0 & 0 & 0
\end{array}, \quad e_{52}=\begin{array}{rrr}
0 & 1 & 0 \\
0 & 0 & 0 \\
0 & -1 & 0
\end{array}, \\
& e_{51}^{\prime}=\begin{array}{rrr}
-1 & 0 & 1 \\
0 & 0 & 0 \\
-1 & 0 & 1
\end{array}, \quad e_{52}^{\prime}=\begin{array}{rrr}
1 & 0 & 1 \\
0 & 0 & 0 \\
-1 & 0 & -1
\end{array} .
\end{aligned}
$$

Taking as $D$ the operator of figure 1, we get

$$
\begin{aligned}
& D e_{51}=-4 e_{51}+e_{51}^{\prime} \\
& D e_{51}^{\prime}=2 e_{51}-4 e_{51}^{\prime} .
\end{aligned}
$$

The functions $e_{52}, e_{52}^{\prime}$ are transformed in the same way.

The eigenvalue problem of the operator $D$ with vanishing boundary values gives the equation

$$
D f+\lambda f=0 .
$$

Let us put $f=a e_{51}+b e_{51}^{\prime}$. Then (10) yields the eigenvalue problem

$$
\begin{aligned}
& (\lambda-4) a+2 b=0 \\
& a+(\lambda-4) b=0 .
\end{aligned}
$$

And from this follow the eigenvalues

$$
\lambda=4 \pm \sqrt{2}
$$

of the operator $D$, each of them having at least the multiplicity 2 .

It is not too hard to establish for a lattice in a given region $A$ the rectangles of base vectors when we know the characters and the irreducible representations of the symmetry group of $A$. It is convenient to choose all those vectors (their total number is equal to the number of lattice points) orthogonal to each other. This is possible because the representation $R$ is orthogonal, and therefore the irreducible subspaces of $S$ are orthogonal.

At the Institute of Applied Mathematics in Zurich (Switzerland) we used the methods of this paper in order to solve the boundary-value problem of Airy's elasticity function inside a dam. As subregions in Schwarz's alternating process, we chose squares of 16 lattice points.

In his book about eigenvalue problems, L. Collatz introduces the notion of "eigenvalues of a graph" and discusses an example, where the graph is built by the segments joining two midpoints of edges of a cube. This problem may also be treated

${ }^{5}$ L. Collatz, Eigenwertaufgaben mit technischen Anwendungen, n. 832 (Leipzig, 1949). by characters, using the group of all rotations of the cube.

In this section another application of character theory is discussed, related to the methods of S. Bergman and M. Picone for the solution of boundary-value problems. For our purposes those methods may be characterized by the following procedure. At first a complete set of particular solutions of the given partial differential equation is constructed and then the solution of the boundaryvalue problem is expanded into a series having as terms those particular integrals. Let us take, for instance, Laplace's equation $\Delta u=0$ in three-dimensional space. The particular solutions may be in this case the harmonic polynomials of degree $n$

$Y_{n}^{(m)}(x, y, z)=r^{n} \cdot e^{m i \phi} \cdot P_{n}^{(m)}(\cos \theta), \quad n=0, i, 2, \ldots$

$m$ running from $(-n)$ to $(+n)$. On the right side, $r, \theta, \phi$ are polar coordinates, $P_{n}^{(m)}$ is a generalized Legendre polynomial. Suppose now that the fundamental region $A$ of the boundary-value problem is a cube having its center at the origin and suppose furthermore that the given boundary values are invariant under the group $G$ of the 24 rotations of the cube. It is obvious that under those circumstances only harmonic polynomials appear in the

\begin{tabular}{|c|c|c|}
\hline Class & Typical element & $\begin{array}{l}\text { Number } \\
\text { of ele- } \\
\text { ments }\end{array}$ \\
\hline $\begin{array}{l}{[1]_{-}} \\
{[2]} \\
{[3]_{-}} \\
{[4]_{--}} \\
{[5]_{-}}\end{array}$ & $\begin{array}{l}\text { Identity } \\
\text { Rotation } \pm 90^{\circ} \\
\text { Rotation } 180^{\circ}(z \text {-axis }) \\
\text { Rotation } 180^{\circ}(\text { axis: } z=0, x=y) \\
\text { Rotation } \pm 120^{\circ} \text { (axis= diagonal) }\end{array}$ & $\begin{array}{l}1 \\
6 \\
3 \\
6 \\
8\end{array}$ \\
\hline & Total $\ldots$ & 24 \\
\hline
\end{tabular}
Bergman-Picone expansion, which have the same symmetry properties. In other words, we have the problem to establish all harmonic polynomials invariant under the group $G$ of rotations of the cube. ${ }^{6}$

The theory of characters gives general methods in order to solve problems of this type. $G$ has the following similarity classes:

By any rotation of the cube the $(2 n+1)$ harmonic polynomials of a given degree $n$ are transformed linearly among themselves and yield therefore a representation $R_{n}$ of degree $(2 n+1)$ of $G$. Let $M$ be any rotation of $G$ and $\chi_{n}(M)$ the character of $R_{n}$. The number of linear independent harmonic polynomials of degree $n$ invariant under $G$ is equal to the multiplicity $c_{n}$ of the unit representation in the decomposition of $R_{n}$ into irreducible components. (The unit representation of a group maps every

6 This question has been posed and answered by G. Polva and B. Mever, Sur les svmétries des fonctions sphériques de Laplace, Compt. rend. 228, 28-30 (1949); Sur les fonctions sphérioues de Laplace de symétrie cristallograpr rique donnée, Compt. rend. 225, (1949). 
group element into the one-row matrix $=1$ ). This multiplicity is given by the character formula

$$
c_{n}=\frac{1}{24} \sum_{(M)} \chi_{n}(M)
$$

$M$ running through the group. It remains to compute the character $\chi_{n}(M)$. In order to do this we may restrict ourselves to rotations $M$ around the z-axis. Let $\mu$ be the rotation angle. Taking into account (14), it follows that the $(2 n+1)$ harmonic polynomials of degree $n$ are transformed by $M$ in the following way:

$$
Y_{n}^{(m)} \rightarrow e^{m i \mu} Y_{n}^{(m)} .
$$

The matrix of the transformation is pure diagonal with the trace

$$
\chi_{n}(M)=\sum_{m=-n}^{+n} e^{m i \mu}=\frac{\sin (2 n+1) \frac{\mu}{2}}{\sin \frac{\mu}{2}} .
$$

From (16) and the table (15) follows now the result:

$$
\begin{aligned}
c_{n} & =\frac{1}{24}\left\{(2 n+1)+6 \frac{\sin (2 n+1) 45^{\circ}}{\sin 45^{\circ}}\right. \\
& \left.+9 \sin (2 n+1) 90^{\circ}+8 \frac{\sin (2 n+1) 60^{\circ}}{\sin 60^{\circ}}\right\} .
\end{aligned}
$$

The final result may be expressed in the following terms. Write $n$ in the form of a multiple of 12 and the corresponding remainder

$n=12 \cdot k+r, \quad 0 \leq r<12$.

Then

$$
c_{n}=k+t(r),
$$

where $t(r)$ is given by the table

\begin{tabular}{|c|rrrrrrrrrrrr|}
\hline$r$ & 0 & 1 & 2 & 3 & 4 & 5 & 6 & 7 & 8 & 9 & 10 & 11 \\
$t(r)$ & 1 & 0 & 0 & 0 & 1 & 0 & 1 & 0 & 1 & 1 & 1 & 0
\end{tabular}.

The polynomials themselves can be built by choosing any harmonic polynomial of degree $n$, applying the 24 rotations of the cube and adding up the 24 resulting polynomials.

A more general problem would be to establish the harmonic polynomials being transformed by $G$ according to a given irreducible representation of $G$. This can be used to simplify the solution of a boundary-value problem in which the given boundaryvalues have not the symmetry of the cube. The basic idea is, of course, the same as outlined previously in this paper.

Los Angeles, January 29, 1952. 